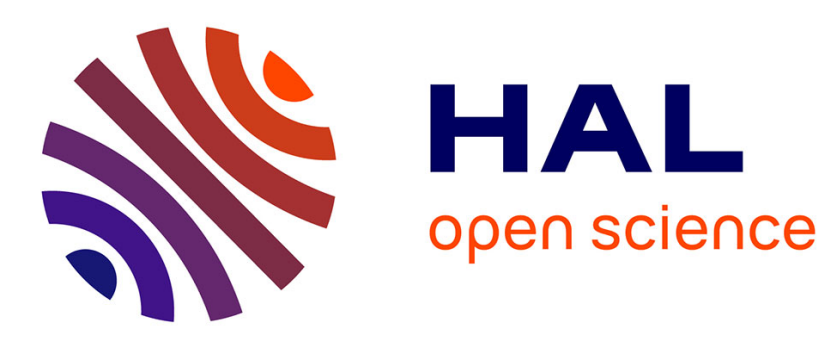

\title{
SÉRIES DE DEBYE ET INTERFÉRENCES DANS UNE PLAQUE ÉLASTIQUE
}

\author{
J. Conoir
}

\section{To cite this version:}

J. Conoir. SÉRIES DE DEBYE ET INTERFÉRENCES DANS UNE PLAQUE ÉLASTIQUE. Journal de Physique IV Proceedings, 1992, 02 (C1), pp.C1-695-C1-704. 10.1051/jp4:19921151 . jpa-00251110

\section{HAL Id: jpa-00251110 https://hal.science/jpa-00251110}

Submitted on 1 Jan 1992

HAL is a multi-disciplinary open access archive for the deposit and dissemination of scientific research documents, whether they are published or not. The documents may come from teaching and research institutions in France or abroad, or from public or private research centers.
L'archive ouverte pluridisciplinaire HAL, est destinée au dépôt et à la diffusion de documents scientifiques de niveau recherche, publiés ou non, émanant des établissements d'enseignement et de recherche français ou étrangers, des laboratoires publics ou privés. 


\title{
SERIES DE DEBYE ET INTERFÉRENCES DANS UNE PLAQUE ELASTIQUE
}

\author{
J.M. CONOIR \\ Le Centre Thomson d'Applications Radars, 6 nue Nieuport,BP. 16, F-78143 Vélizy-Villacoublay cedex, \\ France
}

\begin{abstract}
Résumé
Nous présentons l'essentiel des travaux effectués ces dernières années sur les séries de Debye. Effectuer une décomposition en série de Debye consiste d'une manière générale à écrire l'interaction globale d'une onde avec un diffuseur comme une suite d'interactions locales. Appliquée à la plaque élastique, nous montrons alors le lien profond existant entre les résonances et les interférences. Plus précisément, nous montrons que les résonances observées aux croisements des courbes de dispersion des ondes de Lamb symétriques et antisymétriques sont dues aux interférences entre toutes les ondes longitudinales et transversales multiplement réfléchies dans la plaque.
\end{abstract}

\begin{abstract}
We describe the main works of these last years about the Debye's séries. These last ones provide in a general way with an expansion of the total interaction between a wave and a scatterer into some local interactions. Applied to an elastic plate, we explain the close relation between resonances and interferences. More accurately, we show that observed resonances when the dispersion curves of symmetric and antisymmetric Lamb waves are crossing, result from interferences between all the longitudinal and transversal waves refracted into the plate.
\end{abstract}

\section{Introduction}

L'étude du champ diffusé par un objet élastique, immergé dans l'eau, lorsque celui-ci est insoné par une onde de pression, constitue le problème de la diffusion acoustique. Pour les diffuseurs de forme géométrique dite canonique (cylindre, sphère, plan,...) il existe des solutions analytiques exactes décrivant la diffusion : cellesci sont données sous forme de séries de modes normaux de vibration, ou séries de Rayleigh. Il faut alors remarquer que les séries modales sont des exemples typiques où le modèle mathématique est bien l'image globale de la situation physique examinée, mais où, en même temps, il dissimule la plupart des caractéristiques fines qui pourraient être observées dans le processus de diffusion. Ainsi, par exemple, la série modale ne rend pas compte, à première vue, des multiples réflexions et réfractions géométriques dont le diffuseur est le siège (cf. figure).

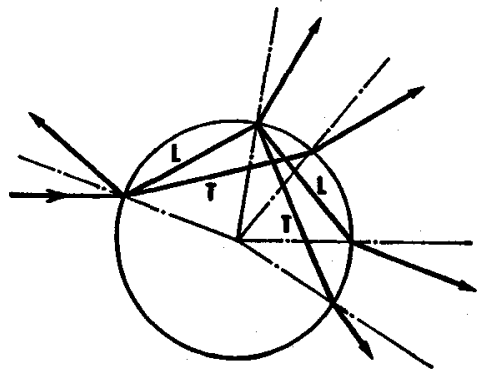


C'est précisément pour cette raison que la série de Debye a été introduite, car elle permet, dans son principe, de décomposer une interaction dite globale en une suite d'interactions locales, et donc de faire apparaître les réflexions et réfractions mentionnées précédemment.

L'intuition qui amène à parler d'interaction locale, comme celle observée au passage d'un dioptre plan, repose habituellement sur l'emploi des ondes géométriques. Or, dès l'instant où la théorie doit être développée au niveau modal, les ondes géométriques n'apparaissent plus immédiatement, d'où une difficulté. Ce problème a été résolu en postulant qu'une interaction dite locale, au niveau modal, doit obéir aux mêmes lois qu'une interaction locale attachée aux ondes géométriques. A savoir qu'une onde (cylindrique, sphérique,...) rencontrant une interface crée, selon que les milieux sont fluides ou élastiques, une ou deux ondes réfléchies et une ou deux ondes réfractées qui se propagent en s'éloignant de l'interface.

On introduit alors les ondes entrantes et sortantes en faisant intervenir la condition de rayonnement à l'infini de Sommerfeld.

\section{Les séries de Debye à travers le siècle}

En 1908, Debye publie un article dans lequel il s'intéresse à la diffusion d'une onde électromagnétique par un cylindre diélectrique [1] , ce qui correspond en acoustique à la diffusion par un cylindre de type fluide où seules les ondes longitudinales $L$ se propagent. Il fait alors apparaître la réflexion spéculaire, et un terme contenant une fraction en suggérant d'effectuer une décomposition de celle-ci pour obtenir les ondes multiplement réfléchies à l'intérieur du cylindre. C'est la genèse des séries de Debye. Dans leur travail de 1937 sur la sphère diélectrique [2], Van Der Pol et Bremmer procèdent différemment car ils définissent pour la première fois, à partir des modes normaux de vibration, des "coefficients de réflexion et de réfraction sphériques". Ils montrent ensuite, par une transformation intégrale, que ceux-ci peuvent être regardés comme une généralisation des premiers coefficients dus à Fresnel. Ils obtiennent également une série aux propriétés géométriques qu'ils associent aux rayons de l'optique géométrique classique. Les principaux problèmes encore rencontrés par Van Der Pol et Bremmer seront résolus par Franz en 1957 [3]. Celui-ci transforme d'emblé la série des modes normaux de vibration en une intégrale de contour, puis fait intervenir la transformée d'Imaï qui permet de dégager une contribution intégrale pouvant se calculer par la méthode du col. Il montre alors, en se référant à Van Der Pol et Bremmer, que la série de Debye peut être introduite à cet endroit, et que l'intégration des termes successifs s'effectue sans problèmes pour donner la contribution due aux ondes géométriques multiplement réfléchies dans le diffuseur. La procédure suivie par Franz correspond à la transformation đite de Sommerfeld-Watson [4] ; nous en donnons un bref rappel au paragraphe suivant. Dans le domaine de l'acoustique, Nagase [5] et Scholte [6] traitent en 1956 le problème de la sphère fluide incluse dans un espace élastique infini. Ils construisent à cette occasion ce que nous appelons la série de Debye du problème, en suivant une démarche plutôt heuristique. Toujours en électromagnétisme, Nussenzveig tente d'établir une théorie complète de la diffusion par une sphère diélectrique en appliquant la transformation de Sommerfeld-Watson aux termes successifs de la série de Debye [7]. Pour les trois derniers travaux mentionnés, il est important de remarquer que la série de Debye est étudiée directement à partir des modes normaux de vibration, en se servant de la notion d'ondes entrantes et sortantes et en considérant un processus d'interaction locale au niveau de l'interface. En acoustique, Brill et Überall font paraître en 1971 un article important sur les ondes géométriques à l'intérieur d'un cylindre élastique [8]. Cette fois, les ondes transmises (réfractées) dans le diffuseur ne sont plus uniquement de type longitudinal L, mais également de type transversal T, et il apparaît des conversions de mode entre ces deux types d'ondes qui compliquent beaucoup l'écriture de la série de Debye. Du reste, celle-ci est obtenue par un principe de récurrence où les règles physiques ne sont pas toujours claires. Les ondes géométriques sont obtenues en effectuant la transformation de Sommerfeld-Watson, transformation qui est également introduite en 1982 par Derem [9] afin d'étudier les ondes géométriques dans un tube de type fluide. Avec cet auteur, la série de Debye prend la forme d'une fraction continue limitée due à la présence des deux interfaces du tube. Avec ces travaux, principalement celui se rapportant au cylindre élastique, il est apparu nécessaire d'établir à partir de quelles règles physiques la série de Debye doit être construite, particulièrement lorsque le diffuseur est élastique et multicouche. Un formalisme matriciel est élaboré en 1980 par Gérard qui répond à ce difficile problème [10] . L'idée développée consiste à introduire des opérateurs locaux qui décrivent la réflexion-réfraction à chaque interface du diffuseur et à les sommer en suivant les lois de l'acoustique géométrique pour retrouver la diffusion globale. Cette idée a évolué tout en restant dans le même esprit [11], avant d'en arriver en 1985 à la formulation actuelle qui permet d'écrire la série de Debye, pour un diffuseur élastique, en suivant des lois physiques claires [12]. 
Dans cet article de 1985, l'auteur n'introduit plus d'opérateurs locaux à chaque interface, mais postule que l'opérateur global qui décrit l'ensemble de la diffusion contient implicitement toutes les interactions locales. Il montre ensuite que l'opérateur global s'éclate facilement, en suivant des régles opératoires simples, et fait apparaître naturellement toutes les interactions locales et la façon dont elles se combinent en suivant les lois de l'acoustique géométrique. C'est en quelque sorte une démarche inverse à celle précédemment adoptée. Cette manière d'obtenir les séries de Debye est exposée clairement dans un article récent, de 1991, dans lequel la série de Debye est également appliquée à la diffusion par une plaque élastique [13]. Signalons pour conclure qu'une étude des ondes géométriques multiplement réfléchies, dans un cylindre de type fluide, a été effectuée pour une onde incidente oblique [14].

\section{Les ondes géométriques dans un cylindre}

Dans ce paragraphe, nous rappelons brièvement la façon dont la série de Debye s'introduit dans la transformation de Sommerfeld-Watson pour faire apparaître les ondes géométriques. Considérons donc une onde plane incidente, en incidence normale, sur un cylindre de type fluide ou élastique. Si on note $P_{\text {Tot }} l a$ superposition de l'onde incidente avec la pression diffusée, alors :

$$
P_{\text {Tot }}=\sum_{n=0}^{+\infty}(-1)^{n} F_{n}\left(x_{1}\right)
$$

avec

$$
F_{n}\left(x_{1}\right)=\frac{(-i) n}{2}\left(2-\delta_{n o}\right)\left[H_{n}^{(2)}\left(k_{1} r\right)+S_{n}\left(x_{1}\right) H_{n}^{(1)}\left(k_{1} r\right)\right] \cos n \phi
$$

où $x_{1}=k_{1} a\left(k_{1}:\right.$ nombre $d^{\prime}$ onde dans le fluide environnant, $a$ : rayon du cylindre) est la fréquence réduite et $S_{n}\left(x_{1}\right)$ la $n$-ième valeur propre de la matrice de diffusion [15]. L'expression (1) est celle de la série des modes normaux de vibration et $(r, \phi)$ sont les coordonnées cylindriques. Avec la convention de temps $e^{-i \omega t}$, les ondes $\mathrm{H}_{\mathrm{n}}^{(1)}\left(\mathrm{H}_{\mathrm{n}}^{(2)}\right)$ sont sortantes (entrantes).

Effectuer la transformation de Sommerfeld-Watson consiste d'abord à remplacer la série des modes normaux par une intégrale de contour dans le plan complexe $\nu$, où $\nu$ est le prolongement analytique sur les modes de vibration $\mathbf{n}$. Il vient ainsi

$$
P_{T o t}=\frac{i}{2} \int_{c} \frac{d \nu}{\sin \pi \nu} F_{\nu}\left(x_{1}\right)
$$

avec le contour ci-dessous :

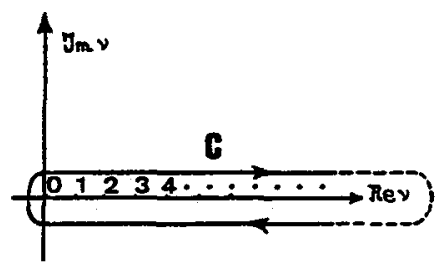

Elle consiste ensuite à déformer ce contour de manière à entourer les singularités physiquement intéressantes de $F_{\nu}\left(x_{1}\right)$, qui sont des pôles de $S_{\nu}\left(x_{1}\right)$, ce qui fournit la partie de la diffusion due au rayonnement des ondes de surface calculée en utilisant le théorème des résidus. Au cours de la déformation du contour, il est nécessaire de distinguer parmi toutes les ondes de surface, celles qui doivent d'abord faire le tour du diffuseur avant de rayonner vers l'observateur [4]. 
$C^{\prime}$ est pourquoi il faut introduire judicieusement la transformation d'Imaï

$$
i \frac{\cos \nu \phi}{\sin \pi \nu}=e^{i \nu(\pi-\phi)}+i e^{i \nu \pi} \frac{\cos \nu(\pi-\phi)}{\sin \pi \nu},
$$

ce qui conduit à isoler, à partir du premier terme du second membre de (4), l'intégrale de contour attachée aux ondes géométriques

$$
P_{\text {géom }}=\int_{c_{g \in a m}} e^{i\left(\frac{\pi}{2}-\phi\right) \nu} S_{\nu}\left(x_{1}\right) H_{\nu}^{(1)}\left(k_{1} r\right) d \nu .
$$

C'est dans cette intégrale que la série de Debye est introduite. Considérons par exemple la série de Debye du cylindre fluide [14], qui est une expression de la forme

$$
S_{\nu}\left(x_{1}\right)=R_{12}(\nu)+\sum_{n=0}^{+\infty} T_{12}(\nu) R_{21}^{n}(\nu) T_{21}(\nu)
$$

Quand on l'introduit dans (5), il vient :

$$
P_{\text {geom }}=\frac{1}{2} \int_{c_{g \epsilon o m}} e^{i\left(\frac{\pi}{2}-\phi\right) \nu} R_{12}(\nu) H_{\nu}^{(1)}\left(k_{1} r\right) d \nu+\ldots
$$

ce qui fait apparaître, chaque intégrale étant évaluée par la méthode du col, les ondes qui sont multiplement réfléchies dans le diffuseur avant de rayonner dans le fluide environnant (cf. figure).

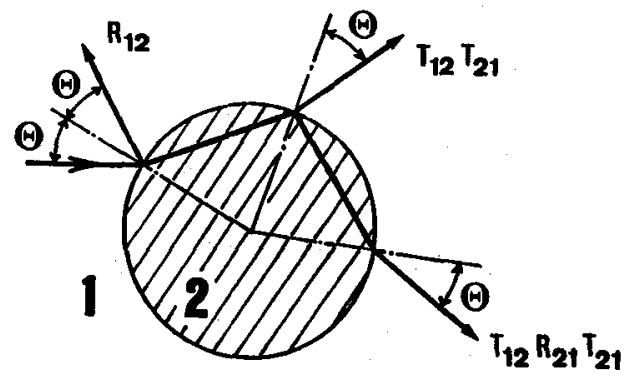

Lorsqu'on calcule les intégrales de (7) par la méthode du col, les coefficients de réflexion-réfraction (transmission) sont obtenus, à un terme de phase près, attaché au trajet acoustique suivi par l'onde, en prenant les expressions de $R_{12}, T_{12}, \ldots$, au col $\nu_{g}=x_{1} \sin \theta$. Les coefficients deviennent alors des fonctions de l'angle d'incidence $\theta$, et on passe ainsi à l'acoustique géométrique. Il faut alors remarquer que les coefficients obtenus sont définis à partir des fonctions de Hankel, liées à la géométrie cylindrique, ce qui en fait des "coefficients de réflexion-réfraction cylindriques" qui tiennent compte de la courbure du cylindre. On montre ensuite, sous l'hypothèse a --> +o (a : rayon du cylindre), que ces coefficients tendent vers ceux bien connus décrivant la réflexion-réfraction à une interface plane, bien que certaines clarifications soient encore nécessaires [14]. C'est en ce sens qu'ils sont une généralisation des premiers coefficients de Fresnel.

\section{La série de Debye modale}

La série de Debye est cherchée, suivant les auteurs, au niveau modal ou au niveau de l'acoustique géométrique, c'est-à-dire avant ou après que soit effectuée la transformation de Sommerfeld-Watson. Cela n'a pas une grande importance dans la mesure où l'on passe de la série de Debye modale à celle de l'acoustique géométrique par un simple prolongement analytique $(n \rightarrow \nu)$, qui est une opération formelle vis-à-vis des manipulations algébriques mises en oeuvre pour obtenir ces séries. 
Les séries de Debye ont été historiquement introduites pour l'étude des ondes géométriques et l'essentiel des travaux publiés se rapportent à cette question. Cependant, la décomposition en série de Debye au niveau modal a également son intérêt puisqu'on s'en est déjà servi afin de chercher un fond potentiel à la matrice de diffusion [16]. Le problème à résoudre est alors le suivant. Lorsqu'on cherche les résonances en étudiant les transitions de phase de la matrice de diffusion [17], on doit factoriser celle-ci par un fond, dit potentiel, qui doit correspondre à la partie non résonnante de la diffusion. Habituellement, on considère le fond potentiel qui correspond à la diffusion par la même cible, celle-ci étant supposée rigide (donc non résonnante). Mais il se trouve que dans certaines situations ce choix n'est pas bien adapté [18]; ce qui a conduit à explorer d'autres voies. C'est ainsi qu'il a été introduit, pour le fond potentiel, le "coefficient de réflexion" modal $R_{12}(n)$ de la série de Debye. Ce coefficient modal n'a pas de sens physique simple cependant, il conduit une fois la transformation de Sommerfeld-Watson effectuée au calcul de la réflexion spéculaire géométrique, qui est intuitivement une part importante de la réponse non résonnante de la cible. D'où l'idée que ce coefficient modal doit contenir une part de réflexion pure, au sens de l'acoustique géométrique, même si on ne sait pas exactement sous quelle forme, ce qui en fait un bon candidat comme fond potentiel. L'étude effectuée dans la référence [16] a confirmé cette analyse. Remarquons bien qu'il existe une infinité de coefficients modaux $R_{12}(n)$ qui se transforment en un coefficient de réflexion unique $R_{12}\left(\nu_{g}=x_{1} \sin \theta\right)$, après transformation de SommerfeldWatson. On comprend alors qu'il est difficile de déterminer quelle proportion de la réflexion spéculaire est contenue dans le coefficient modal $R_{12}(n)$ pour un mode $n$ donné. Notons également que $R_{12}(n)$ pourrait peut être contenir une fraction de rayonnement due à une ou plusieurs ondes de surface, mais cette conjecture $n^{\prime} a$ jamais été établie de façon quantitative. On trouve là l'ensemble des problèmes posés lorsqu'on cherche à donner une interprétation physique à certains coefficients modaux.

\section{La série de Debye et la plaque élastique}

Dans la suite, nous noterons $C_{L}$ et $C_{T}$ les célérités longitudinale $L$ et transversale $T$ des ondes dans la plaque, $C_{1}$ la célérité des ondes dans le fluide environnant (le nombre d'onde est noté $k_{1}$ ), $N_{L}=C_{1} / C_{L}$ et $N_{T}=C_{1} / C_{T}$ les indices de réfraction.

Nous allons maintenant examiner de quelle manière les séries de Debye ont permis d'apporter des réponses au problème suivant, à savoir : comment expliquer physiquement la formation des résonances dans une plaque élastique ?

Pour répondre à cette question, il faut au préalable définir ce qu'est une résonance acoustique, ce qui n'est pas complètement évident. Pour qu'il y ait une résonance acoustique il faut une quantification et un phénomène de renforcement. Ainsi, pour un cylindre ou une sphère, on observe un phénomène de résonance lorsqu'une onde de surface élastique boucle en phase avec elle même (renforcement) à la périphérie du diffuseur (quantification) [19]. Pour une plaque, la quantification sera naturellement fixée par son épaisseur, notée $h$, et le renforcement ne peut venir a priori que d'un phénomène d'interférences. Pour se fixer les idées, rappelons que dans le cas d'une lame fluide les résonances s'interprètent par des interférences constructives sur les ondes longitudinales $L$ multiplement réfléchies à l'intérieur de la lame (cf. figure),

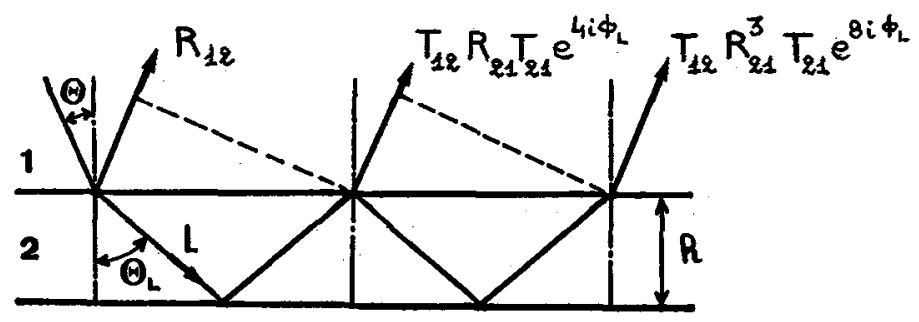


suivant un principe identique à celui d'un interféromètre en optique. Plus précisement, la loi de SnellDescartes s'écrit

$$
\sin \theta=\mathrm{N}_{\mathrm{L}} \sin \theta_{\mathrm{L}}
$$

et la condition d'interférences constructives est réalisée quand tous les rayons ressortent en phase, c'est-à-đire quand :

$$
2 \phi_{L}=\left(k_{1} h\right) N_{L} \cos \theta_{L}=n \pi
$$

$\phi_{\mathrm{L}}$ étant la phase associée au trajet acoustique d'une onde $\mathrm{L}$ entre les deux interfaces de la plaque. Nous pouvons évidemment effectuer une décomposition en série de Debye du coefficient de réflexion (global) de la lame, auquel cas nous faisons apparaître la série des réflexions multiples décrites sur la figure précédente. De plus, l'équation caractéristique de la lame fluide s'écrit alors sous la forme [20]

$$
1-\left(R_{21}\right)^{2} e^{4 i \phi_{L}}=0
$$

qui a pour solution la fréquence réduite complexe $\widehat{\mathbf{k}_{1} \overrightarrow{\mathrm{h}}}$ donnée par la relation

$$
\left(\widetilde{\mathrm{K}_{1} \mathrm{~h}}\right) \mathrm{N}_{\mathrm{L}} \cos \theta_{\mathrm{L}}=\mathrm{n} \pi+\mathrm{i} \log \left|\mathrm{R}_{21}\right| \quad(\mathrm{n} \in \mathrm{N})
$$

où $R_{21}$ est le coefficient de réflexion interne dans la lame. Il convient de remarquer que toutes les équations caractéristiques ayant la même structure que (9) sont susceptibles de conduire à une interprétation des résonances en termes d'interférences.

La situation pour une plaque élastique est évidemment beaucoup plus complexe car il se propage deux types d'ondes L et T. La complexité vient alors des conversions de modes entre ces ondes, conversions qui se produisent chaque fois qu'une onde $L$ ou $T$ rencontre une interface de la plaque. Une étude récente de la série de Debye de la plaque élastique incluant des considérations sur sa convergence et sur les phénomènes énergétiques sous-jacents est effectuée dans la référence [21].

En vue de faire ressortir les phénomènes d'interférences, le premier problème est donc d'écrire l'équation caractéristique de la plaque élastique sous une forme similaire à (9), où il apparaît explicitement les coefficients de réflexion-réfraction locaux à chaque interface, et les phases correspondant aux trajets acoustiques suivis par les ondes $L$ et $T$ qui se propagent dans la plaque. Or, c'est précisément le type même de problème qui est résolu par la série de Debye. Pour ce qui est de la mise en équation en elle-même, nous nous reporterons à la référence [22] où les calculs sont développés en détail. Pour se fixer les idées, précisons que la décomposition en série de Debye conduit à introduire au niveau de chaque interface une suite de 9 coefficients de réflexion-réfraction, afin de décrire localement ce qu'il advient lorsqu'une onde $\mathrm{L}$ ou $\mathrm{T}$ rencontre cette interface. On montre ensuite qu'il existe des relations quadratiques entre ces coefficients, ce qui amène finalement à écrire l'équation caractéristrique de la plaque élastique sous la forme (les fluides de chaque côté de la plaque sont identiques)

$$
S\left(k_{1} h\right)=1-R_{12} e^{2 i\left(\phi_{L}+\phi_{T}\right)}-\left[R_{21}^{L L} e^{2 i \phi_{L}}-R_{21}^{T T} e^{2 i \phi_{T}}\right]=0
$$

pour les modes symétriques, et

$$
A\left(k_{1} h\right)=1-R_{12} e^{2 i\left(\phi_{L}+\phi_{T}\right)}+\left[R_{21}^{L L} e^{2 i \phi_{L}}-R_{21}^{T T} e^{2 i \phi_{T}}\right]=0
$$

pour les modes antisymétriques. Dans ces équations, le terme de phase lié aux ondes $\mathrm{T}$ a pour expression

$$
2 \phi_{\mathrm{T}}=\left(\mathrm{k}_{1} \mathrm{~h}\right) \mathrm{N}_{\mathrm{T}} \cos \theta_{\mathrm{T}}
$$

où $\sin \theta=N_{T} \sin \theta_{T}$, et $R_{12}, R_{21}^{L L}, R_{21}^{T T}$ sont respectivement les coefficients, de réflexion spéculaire, de réflexions internes d'une onde $L$ en une onde $L$ et d'une onde $T$ en une onde $T$. 
Signalons ici un résultat intéressant en soit : l'équation caractéristique écrite sous la forme (10) a un certain caractère intrinsèque, en ce sens qu'elle est invariante, dans sa forme, par rapport aux modifications des conditions de continuité imposées aux deux interfaces de la plaque. Autrement dit, elle s'écrit toujours de la même façon, et lorsque les conditions de continuité changent, ce sont seulement les expressions des coefficients de réflexion aux interfaces qui changent [22].

\section{Résonances et interférences dans la plaque élastique}

Dans le domaine angulaire difini $\operatorname{par} \sin \theta<\mathrm{N}_{\mathrm{L}}$ les ondes $\mathrm{L}$ et $\mathrm{T}$. sont propagatives de sorte que les phases $\phi_{\mathrm{L}}$ et $\phi_{\mathrm{T}}$ sont purement réelles. Par suite, les fonctions $S$ et $A$ de $(10)$ sont presque périodiques en fréquence, ce qui ne permet pas, comme dans le cas fluide, de trouver une interprétation simple des résonances en termes d'interférences, dans l'hypothèse où elle existe. C'est pourquoi nous avons forcé l'équation (10) à ne dépendre que d'une seule période en imposant la condition

$$
\frac{2 i \phi_{\mathrm{L}}}{2 i \phi_{\mathrm{T}}}=\frac{N_{\mathrm{L}} \cos \theta_{\mathrm{L}}}{N_{\mathrm{T}} \cos \theta_{\mathrm{T}}}=\frac{\mathrm{p}}{\mathrm{q}} \quad(\mathrm{p}<\mathrm{q})
$$

où $\mathrm{p}$ et $\mathrm{q}$ sont des entiers. Avec cette condition, il est facile de voir que l'équation (10) a une structure semblable à (9) [22].

La relation (12) impose évidemment une restriction puisque celle-ci est vérifiée uniquement pour une infinité discrète d'angles $\theta$ pq qui ont pour valeur

$$
\sin \theta \mathrm{pq}=\left[\frac{\mathrm{N}_{\mathrm{L}}^{2}-\left(\frac{\mathrm{p}}{\mathrm{q}}\right)^{2} \mathrm{~N}_{\mathrm{T}}^{2}}{1-\left(\frac{\mathrm{p}}{\mathrm{q}}\right)^{2}}\right]^{\frac{1}{2}} \quad\left(\mathrm{pN}_{\mathrm{T}}<\mathrm{qN}_{\mathrm{L}}\right)
$$

On montre alors le résultat important suivant [22] : les courbes de dispersion des ondes de Lamb symétriques et antisymétriques se croisent précisément aux angles définis par (13). On peut observer ces croisements sur la figure ci-dessous représentant les courbes de dispersion d'une plaque en laiton.

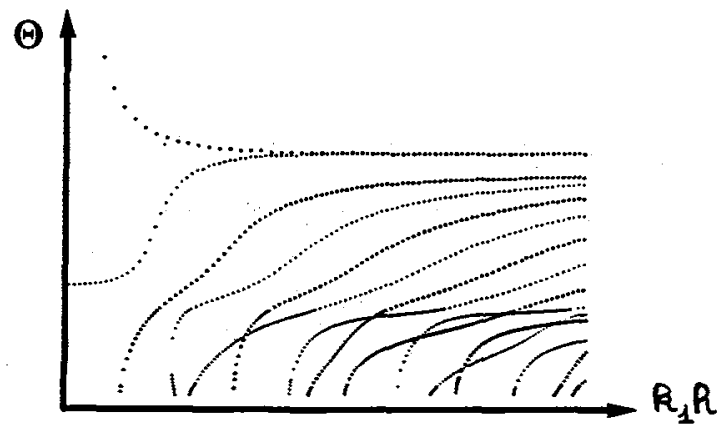

Ce point étant démontré, écrivons maintenant que les ondes multiplement réfléchies, composées des seules ondes L, créent des interférences constructives ; ceci se traduit classiquement par la relation (n est un entier naturel)

$$
\left(k_{1} h\right) N_{L} \cos \theta_{L}=n(p \pi)
$$


Il résulte alors directement de (12) que les ondes multiplement réfléchies, composées des seules ondes T, créent également des interférences constructives puisque

$$
\left(\mathrm{k}_{1} \mathrm{~h}\right) \mathrm{N}_{\mathrm{T}} \cos \theta_{\mathrm{T}}=\mathrm{n}(\mathrm{q} \pi)
$$

Il est ensuite aisé de vérifier que toutes les ondes multiplement réfléchies pour lesquelles on observe des conversions de mode entre les ondes $\mathrm{L}$ et $\mathrm{T}$ sont également en interférences. Par conséquent, à toutes les fréquences données par (14), ou (15), nous observons l'existance dans la plaque élastique de ce que nous appelons un régime d'interférences. Ceci étant acquis, nous avons examiné, pour les angles $\theta$ pq, les fréquences de résonances de la plaque élastique. Nous obtenons alors le résultat remarquable suivant : lorsqu'une fréquence de résonance est observée simultanément dans un mode symétrique et antisymétrique, il existe un entier $n$, tel que cette fréquence soit calculée exactement par la formule (14) ou (15). Ce qui montre que toutes les fréquences, où nous observons un croisement des courbes de dispersion associées aux ondes de Lamb symétriques et antisymétriques, correspondent exactement à des fréquences pour lesquelles nous observons un régime d'interférences dans la plaque élastique. De plus, mais nous n'avons pas réussi à le démontrer clairement, il semble que les seules situations où les résonances s'expliquent par des interférences soient précisément celles observées à l'endroit des croisements des courbes de dispersion, et pas ailleurs.

Afin d'illustrer ce propos, nous avons tracé le comportement de la fonction $S\left(k_{1} h\right)$ en fonction de la fréquence réduite $k_{1} h$. Les deux courbes ci-dessous, où ( $\operatorname{Re} S\left(k_{1} h\right), \operatorname{Im} S\left(k_{1} h\right)$ ) varie en fonction de $k_{1} h$, illustrent parfaitement la situation générale. Ou $\theta$ est différent des angles $\theta \mathrm{pq}$, et la courbe paramétrée a un comportement non régulier (si $k_{1} \mathrm{~h} \rightarrow+\infty$, elle noircit une surface bornée)

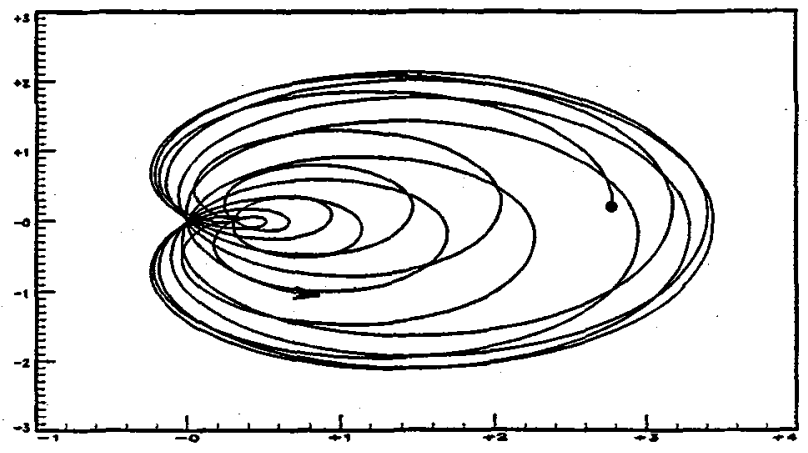

ou $\theta=\theta p q$, et la courbe paramétrée est périodique (dans l'exemple ci-dessous $p=2$ et $q=5$ )

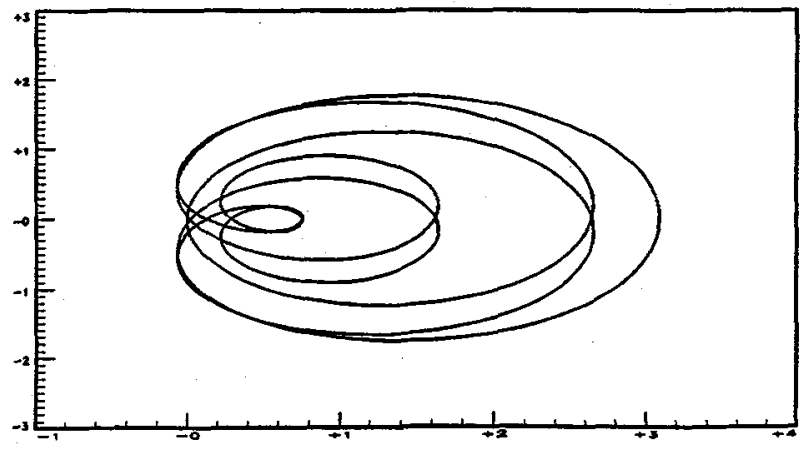

Si on admet qu'en l'absence de périodicité il n'y a pas de phénomène d'interférences, cela tend à montrer que les angles $\theta$ pq sont les seuls pour lesquels on peut observer des interférences. 
Au vu des courbes obtenues aux angles $\theta$ pq, il est légitime de se demander si on observe également une périodicité sur la répartition dès résonances. Pour examiner ce problème, nous devons étudier les solutions de l'équation caractéristique pour les angles $\theta \mathrm{pq}$, c'est-à-dire lorsque (12) est vérifiée. A cette fin nous posons

$$
X=e^{2 i \phi_{L}} \quad \text { et } \quad Y=e^{2 i \phi_{T}}
$$

et avec ces notations, on montre que les équations (10) se transforment en équations polynomiales qui s'écrivent

$$
\left(1+R_{21}^{T T} Y\right)^{q}=Y^{p}\left(R_{12} Y+R_{21}^{L L}\right)^{q} \text { ou }\left(1-R_{21}^{L L} X\right)^{p}=X^{q}\left(R_{12} X-R_{21}^{T T}\right)^{p}
$$

pour les modes symétriques, et

$$
\left(1+R_{21}^{L L} X\right)^{p}=X^{q}\left(R_{12} X+R_{21}^{T T}\right)^{p} \text { ou }\left(1-R_{21}^{T T} Y\right)^{q}=Y^{p}\left(R_{12} Y-R_{21}^{L L}\right)^{q}
$$

pour les modes antisymétriques.

Continuons notre étude avec les ondes symétriques. Comme les polynômes sont de degrés $p+q$, ils ont $p+q$ racines complexes. Soit $z_{1}$ une racine de (17-1), il lui correspond de façon biunivoque une racine $z_{2}$ de (17-2) qui vérifie l'égalité $\mathrm{z}_{1}^{\mathrm{p}}=\mathrm{z}_{2}^{\mathrm{q}}$. Les racines dépendent uniquement de l'angle $\theta$ et des paramètres élastiques de la plaque et du fluide. Par suite, il résulte de (16) que toutes les solutions de (10-S), qu'on note $\left(k_{1} h\right)_{n}$ (n est un entier), ont pour expression à l'angle $\theta \mathrm{pq}$

$$
\text { ou } \begin{aligned}
& \left(k_{1} h\right)_{n} N_{T} \cos \theta_{T}=2 n q \pi+\operatorname{argz} z_{1}-i \log \left|z_{1}\right| \\
& \left(k_{1} h\right)_{n} N_{L} \cos \theta_{L}=2 n p \pi+\arg z_{2}-i \log \left|z_{2}\right|
\end{aligned}
$$

où $z_{1}\left(z_{2}\right)$ est une racine de $(17-1)((17-2))$. Il en résulte alors immédiatement que

$$
\mathrm{T}_{\mathrm{pq}}=\frac{2 \mathrm{q} \pi}{\mathrm{N}_{\mathrm{T}} \cos \theta_{\mathrm{T}}}=\frac{2 \mathrm{p} \pi}{\mathrm{N}_{\mathrm{L}} \cos \theta_{\mathrm{L}}}=2\left[\frac{\mathrm{q}^{2}-\mathrm{p}^{2}}{\mathrm{~N}_{\mathrm{T}}^{2}-\mathrm{N}_{\mathrm{L}}^{2}}\right]^{\frac{1}{2}} \pi
$$

est une période de répétition des résonances. Plus précisément, nous avons un nombre fini de racines $z_{i}$ solutions de (17) qui se répètent à la période $T_{\mathrm{pq}}$.

\section{Conclusion}

Les séries de Debye ont été initialement introduites pour faire apparaître et étudier les ondes géométriques qui se propagent dans un diffuseur, et ce n'est qu'assez récemment qu'on s'est demandé si elles avaient un champ d'application plus vaste. C'est ainsi qu'est ressortie l'idée qu'une décomposition en série de Debye consiste, en fait, à décrire une interaction globale comme une suite d'interactions locales. Après quoi, la série de Debye a été utilisée à d'autres fins, et c'est ainsi par exemple qu'on a pu apporter des réponses au problème du choix $d^{\prime} u n$ fond potentiel pour la matrice de diffusion, et su établir le lien profond entre les résonances et les interférences dans une plaque élastique. Nous pensons d'une manière générale que le fait de décomposer une interaction globale en une suite d'interactions locales peut éclairer d'un jour nouveau un grand nombre de problèmes.

\section{Remerciement}

Certains problèmes assez fondamentaux apparaissent de façon sous-jacente dans ce texte, où ils n'ont pu être qu'effleurés, et nous remerçions ici André Derem avec qui les échanges d'idées, suscités par ces problèmes, ont été particulièrement intéressants. 


\section{Bibliographie}

[1] DEBIJE, P, Das elektromagnetische Feld um einen Zylinder und die Theorie des Regenbogens, Phys. Z.9 (1908) 775-778.

[2] VAN DER POL, B, BREMMER, H, The diffraction of electromagnetic waves from an electrical point source round a finitely conducting sphere, with applications to radiotelegraphy and the theory of the rainbow, Phil. Mag. 24 (1937) 141-176, 825-864.

[3] FRANZ, W, Theorie der Beugung Elektromagnetischer Wellen, Springer-Verlag, Berlin (1957).

[4] DEREM, A, Théorie de la matrice $S$ et transformation de Sommerfeld-Watson dans la diffusion acoustique ; in La diffusion acoustique, N. GESPA, Chap.9, CEDOCAR, Paris (1987).

[5] NAGASE, M, Diffraction of elastic waves by a spherical surface, J. Phys. Soc. Jpn. 11 (1956) $279-301$.

[6] SCHOLTE, J.G.J, On seismic waves in a Spherical Earth, Koninklijk Nederlands Meteorologisch Institut, Commun. $n^{\circ} 65$ (La Haye, 1956).

[7] NUSSENZVEIG, H.M, High-frequency scattering by a transparent sphere. 1. Direct reflection and transmission, J. Math. Phys. 10 (1969) 82-124; II. Theory of the rainbow and the glory, J. Math. Phys. 10 (1969) 125-176.

[8] BRILL, D, ÜBERALL, H, Acoustic waves transmitted through solid elastic cylinders, J.A.S.A. 50 (1971) 921-939.

[9] DEREM, A, Série des ondes transmises pour un cylindre fluide et creux : une solution exacte, Rev. CETHEDEC. 73 (1982) 1-27.

[10] GERARD, A, Champ résultant de l'incidence d'ondes $P$ et $S V$ sur un milieu stratifié à symétrie sphérique, C.R.A.S. Paris. 290 (1980) 43-46.

[11] GÉRARD, A, Factorisation de l'équation caractéristique d'une sphère élastique multicouches : interprétation des résonances, C.R.A.S. Paris. Série II.297 (1983) 17-19.

[12] CONOIR, J.M., Diffusion acoustique en géométrie séparable : une méthode d'acquisition des séries de Debye généralisées, C.R.A.S Paris. Série II. 301 (1985) 471-473.

[13] CONOIR, J.M, GÉRARD, A, DEREM, A, Ondes acoustiques transmises et séries de Debye généralisées. I. Traitement des interfaces planes, J. Acoustique. 4 (1991) 159-200.

[14] CONOIR, J.M, Diffusion acoustique par un cylindre. Théorie modale et approximation de l'acoustique géométrique, Thèse de l’Université Paris VI (1987).

[15] FLAX, L, DRAGONETTE, L.R, ÜBERALL, H, Theory of elastic resonance excitation by sound scattering, J.A.S.A. 63 (1978) 723-731.

[16] CONOIR, J.M, GERARD, A, Un nouveau fond potentiel pour la matrice S, J. Acoustique. 2 (1989) 217227.

[17] MURPHY, J.D, BREITENBACH, E.D, UBERALL, H, Resonance scattering of acoustic waves from cylindrical shells, J.A.S.A. 64 (1978) 677-683.

[18] DEREM, A, Diffusion acoustique par un manchon cylindrique et définition du fond non résonnant, Rev. CETHEDEC. 64 (1980) 1-11.

[19] ÜBERALL, H, DRAGONETTE, L.R, FLAX, L, Relation between creeping waves and normal modes of vibration of a curved body, J.A.S.A. 61 (1977) 711-715.

DEREM, A, Relation entre la formation des ondes de surface et l'apparition de résonances dans la diffusion acoustique, Rev. CETHEDEC. 58. (1979) 43-79.

[20] CONOIR, J.M, Réflexion et Transmission par une plaque fluide ; in La diffusion acoustique, N. GESPA, Chap. 5, CEDOCAR, Paris (1987).

[21] DESCHAMPS, M, CAO, C, Reflection-refraction of a solid layer by Debye's serie expansion, sous presse, Ultrasonics.

[22] CONOIR, J.M, Interférences et périodicités sur les résonances dans une plaque élastique, J. Acoustique, 4 (1991) 377-412. 